\title{
Avaliação do banco de germoplasma de mexericas com relação às características físico-químicas e suscetibilidade à mancha marrom de alternária
}

\author{
Marinês Bastianel (*); Lilian Massaro Simonetti; Evandro Henrique Schinor; Ronald Otto De Giorgi; \\ José Dagoberto De Negri; Debir Naves Gomes; Fernando Alves de Azevedo \\ Instituto Agronômico - IAC, Centro de Citricultura Sylvio Moreira/APTA, Caixa Postal 04, 13490-970 Cordeirópolis (SP), Brasil. \\ (*) Autora correspondente: mbastianel@centrodecitricultura.br
}

Recebido: 15/jul./2013; Aceito: 5/fev./2014

\begin{abstract}
Resumo
A produção de tangerinas alcança expressão econômica em alguns estados brasileiros, principalmente em São Paulo, Paraná, Rio Grande do Sul e Minas Gerais. Nos últimos anos, um interesse crescente pelas mexericas tem sido observado no estado de São Paulo, possivelmente pela inviabilidade econômica de manterem-se pomares das principais variedades comerciais para frutos de mesa, tangerina Ponkan e tangor Murcott, devido à alta suscetibilidade à mancha marrom de alternária (MMA). O objetivo deste trabalho foi caracterizar 33 acessos de mexericas do Banco Ativo de Germoplasma de Citros do Instituto Agronômico de Campinas (BAG Citros IAC), visando selecionar variedades com boas características físico-químicas como massa, altura e largura de frutos, cor da casca, rendimento de suco, acidez, sólidos solúveis e Ratio e resistência à MMA para que possam ser comercializadas em outros períodos do ano, em comparação com a variedade mexerica Rio IAC 194, padrão desse grupo, cuja maturação é de meia-estação. Os resultados demonstraram que cerca de 85\% dos acessos de mexerica avaliados em campo e in vitro apresentaram resistência à MMA. Selecionaram-se cinco acessos precoces e 11 mais tardios que a Rio IAC 194 como boas alternativas para a ampliação do período de safra de mexericas no estado de São Paulo.
\end{abstract}

Palavras-chave: Citrus deliciosa, Alternaria alternata, resistência a doenças.

\section{Evaluation of willowleaf mandarin germplasm with respect to characteristics physical, chemical and susceptibility for alternaria brown spot}

\begin{abstract}
The mandarin production achieves economic expression in some Brazilian states, mainly in São Paulo, Paraná, Rio Grande do Sul and Minas Gerais. In recent years a growing interest in these mandarins has been observed in São Paulo, possibly due to the economic viability of maintaining the orchards of the main commercial varieties, Ponkan mandarin and Murcott tangor, due to high susceptibility to alternaria brown spot (ABS). The objective of this study was to characterize 33 accessions of willowleaf mandarins to 'Banco Ativo de Germoplasma de Citros' of the Instituto Agronômico de Campinas ('BAG Citrus IAC'), to select varieties with good horticultural characteristics as mass, height and width of fruit, skin color, juice content, acidity, soluble solids and Ratio, and tolerance to ABS and that can be marketed in other periods of the year in comparison with the Rio IAC 194 mandarin, standard variety - which ripening is half station. The results showed that to about $85 \%$ of the mandarin accessions evaluated in the field and in vitro were resistant to ABS. Was selected five hits early and later eleven more than Rio IAC 194, which can be good alternatives for the extension of the period of harvest of mandarins in the State of Sao Paulo.
\end{abstract}

Key words: Citrus deliciosa, Alternaria alternata, disease resistance.

\section{INTRODUÇÃO}

O Brasil destaca-se como um dos maiores produtores mundiais de laranjas, essencialmente destinadas à indústria de suco concentrado. Como frutos para consumo in natura, as tangerinas são muito apreciadas, principalmente pela facilidade de descascar, sabor agradável e teor de vitamina C. A produçâo brasileira de tangerinas, em 2012, foi em torno de um milhão de tonelada, colhida em uma área de aproximadamente 52 mil hectares nas regiôes Sudeste e Sul do país, principalmente em São Paulo, Paraná, Rio Grande do Sul e Minas Gerais (IBGE, 2014).

A produção de tangerinas no Brasil ainda é incipiente, não sendo suficiente para atender a demanda interna. Dentre as variedades mais plantadas, destacam-se a tangerina Ponkan (Citrus reticulata Blanco) e o híbrido tangor 
Murcott [C. reticulata $\mathrm{x}$ C. sinensis (L.) Osbeck], no estado de São Paulo, e as mexericas Rio ou Caí e Montenegrina (C. deliciosa Tenore), nos estados da regiâo Sul do país. Embora plantadas em menor escala no estado de São Paulo, a produção de mexericas merece destaque na regiáo do Vale do Ribeira, por encontrar condiçóes climáticas favoráveis à obtençâo de frutos de boa qualidade físico-química. Por outro lado, tem-se observado um crescente interesse dos produtores por esse grupo de tangerinas nos últimos anos, possivelmente pela inviabilidade econômica de manterem-se pomares de Ponkan e, principalmente, Murcott, devido à alta suscetibilidade à mancha marrom de alternária (MMA), o que tem prejudicado sobremaneira a produção de frutos para consumo in natura (Azevedo et al., 2010; Stuart et al., 2009).

Presente no país desde 2002, a MMA é causada por um patotipo do fungo Alternaria alternata que produz uma toxina seletiva ao hospedeiro (HST - host specific toxins), infectando as tangerinas, principalmente a Dancy $(C$. reticulata Blanco) e seus híbridos, alguns tangores, como Murcott e, em casos raros, pomelos (C. paradisi Macfad.) (Peres et al., 2003; Timmer et al., 2003). A doença constitui hoje um dos principais problemas fitossanitários na cultura das tangerinas e possivelmente contribuiu para a redução de $15,6 \%$ da área colhida e de $16,8 \%$ da produção, entre os anos de 2010 e 2012, no estado de São Paulo (IBGE, 2014).

O grupo das mexericas é também conhecido como o das tangerinas do Mediterrâneo ou Willowleaf. Seus frutos são de tamanho médio (5 a $6 \mathrm{~cm}$ de diâmetro), achatados, de casca fina e aderida (Schwarz, 2009), com boa aceitação no mercado interno e grande potencial para a exportação por possuírem casca mais firme que outras tangerinas, como a Ponkan, por exemplo. Por outro lado, as mexericas apresentam, em geral, um número elevado de sementes, característica pouco desejada para o mercado de frutas frescas. Quando não realizadas práticas culturais de raleio e poda, as plantas apresentam alternância de produçáo (Schwarz, 2009).

Além das doenças, um dos principais problemas da citricultura de mesa está relacionado à oferta e demanda de frutas para o mercado, tanto interno como externo, uma vez que o período de safra da maioria das variedades comerciais se concentra entre maio e julho. A necessidade de variedades precoces e tardias que possam ampliar o período de oferta tem sido uma das mais importantes demandas do setor.

O Centro APTA Citros Sylvio Moreira (CAPTACSM), do Instituto Agronômico de Campinas (IAC), em Cordeirópolis, SP, possui um Banco Ativo de Germoplasma (BAG Citros IAC) com cerca de 40 acessos de mexericas, dentre os quais se propôs selecionar variedades de mexericas com boas características horticulturais e, principalmente, com resistência à MMA que possam ampliar o período de safra das tangerinas para o estado de São Paulo.

\section{MATERIAL E MÉTODOS}

Foram avaliados 33 acessos de mexericas pertencentes ao BAG Citros IAC (Tabela 1). As coleçôes no campo são constituídas por plantas enxertadas em tangerina Cleópatra (Citrus reshni Hort.), que foram estabelecidas entre 1986 e 1989. Os acessos estão agrupados de acordo com a sua espécie e características de produção, com três repetiçóes, plantadas em espaçamento $7,5 \times 5,5 \mathrm{~m}$, numa regiáo endêmica para a doença mancha marrom de alternária. Avaliaram-se características como severidade de MMA em campo (in vivo) e in vitro e características físico-químicas dos frutos. Além das coleçôes mantidas em campo, o BAG Citros IAC está estabelecido em coleçôes teladas compostas de uma planta por acesso, enxertadas em limão Cravo (Citrus limonia Osbeck) e estabelecidas em vasos de $64 \mathrm{~L}$. Seis acessos, cujas plantas foram erradicadas no campo por problemas fitossanitários, foram avaliados apenas quanto à resposta à inoculaçáo de Alternaria alternata in vitro a partir de material coletado nas coleçôes protegidas (Tabela 1).

\section{Avaliação para mancha marrom de alternária}

\section{Avaliação in vivo}

Vinte e sete acessos de mexerica foram avaliados em campo, quantificando-se a severidade da MMA em folhas e brotaçôes novas com auxílio de uma escala de notas ( 0 a 4), onde zero representa ausência de sintomas e 4 representa planta altamente infectada, isso é, que apresentava mais de $50 \%$ das brotaçóes novas secas. Em frutos, a doença foi quantificada com o uso de uma escala diagramática desenvolvida por Renaud et al. (2008). Essa escala de notas varia de 1 a 6 , correspondendo, respectivamente, à áreas lesionadas de $0,1 \%, 1,0 \%, 2,5 \%, 5,0 \%, 11,0 \%$ e $25,0 \%$ da superfície do fruto. A avaliação foi conduzida em uma amostragem de 20 frutos por planta. Duas variedades comerciais, o tangor Murcott e a tangerina Dancy, também foram avaliadas como padróes de suscetibilidade.

\section{Avaliação in vitro}

No ensaio in vitro a inoculação de $A$. alternata foi realizada em folhas destacadas de 33 acessos de mexerica e mantidas em placas de Petri em temperatura de $27^{\circ} \mathrm{C}$, segundo metodologia modificada por Azevedo et al. (2010). Também nesse caso utilizaram-se como padróes de suscetibilidade folhas de tangor Murcott e de tangerina Dancy.

A inoculação foi realizada em folhas jovens. Foram avaliadas três repetiçóes (placas) contendo três folhas cada. O inóculo inicial foi obtido a partir de lesões típicas da 
doença, que foram desinfectadas superficialmente por meio de lavagens com etanol $70 \%$, hipoclorito $3 \%$ de cloro ativo e água destilada esterilizada, sendo, então, incubado em meio de cultura BDA (200 g batata, $20 \mathrm{~g}$ dextrose e $15 \mathrm{~g}$ agar $\mathrm{L}^{-1}$ ) acrescido do fungicida carbendazin, para inibir a proliferação de fungos contaminantes, e mantido a $27^{\circ} \mathrm{C}$, sob fotoperíodo de 12 horas.

Após 48 horas de crescimento, o micélio emergente foi transferido para meio de cultura BDA e incubado nas mesmas condições do inóculo inicial. Após uma semana foi confirmada a identidade do isolado por meio de microscopia óptica das estruturas de reprodução assexuada. Em seguida, 50 discos $(5 \mathrm{~mm})$ com crescimento micelial foram transferidos para meio de $\mathrm{CaCO}_{3}$ para indução de esporulaçáo $(30 \mathrm{~g}$ de $\mathrm{CaCO}_{3}, 20 \mathrm{~g}$ de sacarose e $20 \mathrm{~g}$ de Agar $\mathrm{L}^{-1}$ ), seguido da adição de $2 \mathrm{~mL}$ de água destilada esterilizada na superfície do meio de cultura. As placas foram incubadas a $27^{\circ} \mathrm{C}$ por quatro dias sob fotoperíodo de 12 horas.

$\mathrm{Na}$ sequência foram adicionados $10 \mathrm{~mL}$ de água destilada esterilizada na superfície das placas e, com a ajuda de uma espátula estéril, os conídios foram raspados e retirados da superfície do meio, filtrados em duas camadas de gaze estéril e quantificados em Câmara de Neubauer (Marca Kasvi), sendo a suspensão de esporos ajustada para a concentração final de $10^{5}$ conídios $\mathrm{mL}^{-1}$. Em seguida, a suspensão do inóculo foi pulverizada sobre as folhas das diferentes variedades $(2$ $\mathrm{mL}$ folha $\left.{ }^{-1}\right)$. As áreas foliares com sintomas de MMA (\%) foram avaliadas 48 e 72 horas após a inoculação com o fungo.

\section{Avaliações físico-químicas dos frutos}

Foram realizadas três avaliações físico-químicas dos frutos em seu período de colheita, em intervalos de três semanas, no Laboratório de Qualidade e Pós-Colheita do Centro APTA Citros/IAC. Foram colhidos 10 frutos na porção externa da copa, na faixa compreendida entre 1,0 e 2,0 m de altura do solo e em toda a extensão do perímetro da planta, em duas repetiçóes (plantas), que foram utilizados para as análises físico-químicas. Foram realizadas as determinaçôes de altura (A) e largura (L) por leitura direta de cada amostra, com auxílio de uma escala graduada em centímetros e a relação entre A/L. A massa total dos frutos foi obtida, de uma só vez, em uma balança (Filizola). A contagem do número de sementes foi realizada em uma amostra casualizada de cinco frutos por acesso, coletados de duas plantas.

O rendimento de suco foi determinado após esmagamento do fruto na extratora OIC (Organização Internacional Centenário) modelo OTTO 1800 (filtro com diâmetro interno $=26,11 \mathrm{~mm}$; comprimento $=265 \mathrm{~mm}$; furos de diâmetro $=0,6 \mathrm{~mm}$; área de vazão $=20 \%$ ), calculado através da relação massa do suco/massa do fruto e expresso em porcentagem.
O teor de sólidos solúveis foi determinado por leitura direta no refratômetro B\&S, modelo RFM 330 e expresso em ${ }^{\circ}$ Brix. Os dados foram corrigidos pela temperatura e pela acidez do suco.

A acidez foi obtida por titulação de $25 \mathrm{~mL}$ de suco, com uma soluçáo de hidróxido de sódio de normalidade 0,3125 e usando-se fenolftaleína como indicadora (adaptado de Reed et al., 1986). Foi calculada a relação sólidos solúveis/ acidez fazendo-se o valor da acidez igual a 1. Essa relação (Ratio) indica o estádio de maturação dos frutos cítricos. Todas essas análises foram avaliadas e comparadas com a variedade de mexerica Rio IAC 194, mais plantada no estado de São Paulo.

A coloraçáo da casca foi medida no aparelho colorímetro Minolta CR 300 em três pontos na região equatorial dos frutos, calculando-se a média para amostra. Foram avaliados cinco frutos de cada repetição (duas plantas), sendo determinados para cada fruto os valores de $L$ (luminosidade de cor), $a$ (variação de cor de verde a vermelho) e $b$ (variação de cor de azul a amarelo). Com os valores de $L$, $a$ e $b$ calculou-se o índice de cor pela fórmula IC $=(1.000 \times a) /$ $(b \times L)$. Esse índice varia entre, aproximadamente, $-20 \mathrm{e}+20$ (escala de cor de Hunter). Valores entre -7 e +7 expressam as tonalidades, de verde-amarelo ( -7 a 0$)$, amarelo pálido a verde alaranjado (valores próximos de zero) e laranja pálido a laranja intenso $(0 \mathrm{a}+7)$; valores superiores expressam cores mais intensas (Jimenez-Cuesta et al., 1983).

\section{Análises estatísticas}

A variável número de sementes por fruto foi submetida à análise de variância em delineamento de blocos ao acaso, com cinco repetiçôes (frutos). Já as variáveis obtidas nas análises físico-químicas dos frutos e no índice de cor da casca dos frutos foram submetidas à análise de variância em esquema fatorial, em blocos ao acaso, com duas repetiçóes. Todos os dados originais foram transformados em $\sqrt{x}_{\mathrm{x}}+1$ e as médias comparadas pelo teste paramétrico Scott Knott (Scott e Knott, 1974) ao nível de 5\% de significância, calculados por meio do programa estatístico SISVAR (Ferreira, 2008).

\section{RESULTADOS E DISCUSSÃO}

\section{Resistência à mancha marrom de alternária}

A maioria dos acessos de mexericas estudados (85,2\%) foi resistente à mancha marrom de alternária, nas avaliaçôes em campo, sendo que apenas quatro acessos (14,8\%), Pará IAC 193, Revero IAC 195, Pernambucana IAC 580 e Precoce IAC 281, foram suscetíveis, porém com baixo 
Tabela 1. Severidade da mancha marrom de alternária em campo e in vitro (48 e 72 horas de inoculação) e número de sementes em diferentes acessos de mexericas do Banco Ativo de Germoplasma de Citros do IAC (Cordeirópolis, SP, 2010)

\begin{tabular}{|c|c|c|c|c|c|}
\hline \multirow{3}{*}{ Origem } & \multirow{3}{*}{ Nome comum } & \multicolumn{3}{|c|}{ Mancha marrom de alternária } & \multirow{3}{*}{$\mathbf{N}^{\circ}$ de sementes } \\
\hline & & \multirow{2}{*}{$\begin{array}{c}\text { No campo } \\
\text { (Nota) }\end{array}$} & \multicolumn{2}{|c|}{ In vitro (\%) } & \\
\hline & & & $48 \mathrm{~h}$ & $72 \mathrm{~h}$ & \\
\hline CV & Ipanema IAC 192 & - & 0 & 1,11 & - \\
\hline CV & Pará IAC 193 & $0,66^{*}$ & 0 & 7,30 & $14,40 \mathrm{~b}$ \\
\hline $\mathrm{CV}$ & Rio IAC 194 & 0 & 0 & 0 & $15,80 \mathrm{~b}$ \\
\hline CV & Revero IAC 195 & $0,33^{*}$ & 0 & 1,10 & - \\
\hline CV & Willow IAC 196 & 0 & 0 & 0 & - \\
\hline CV & Nobilis IAC 199 & 0 & 0 & 0 & $12,80 \mathrm{~b}$ \\
\hline CV & Pará IAC 203 & 0 & 0 & 6,44 & $22,80 \mathrm{a}$ \\
\hline $\mathrm{CV}$ & Ipanema IAC 208 & 0 & 0 & 0 & $12,20 \mathrm{~b}$ \\
\hline $\mathrm{CN}$ & Paulista IAC 567 & 0 & 0 & 0 & $12,40 \mathrm{~b}$ \\
\hline $\mathrm{CN}$ & Sunwikon IAC 576 & 0 & 0 & 0 & $14,40 \mathrm{~b}$ \\
\hline $\mathrm{CN}$ & 114412 IAC 577 & 0 & 0 & 0 & $21,80 \mathrm{a}$ \\
\hline $\mathrm{CN}$ & Pernambucana IAC 578 & 0 & 0 & 0 & $16,20 \mathrm{~b}$ \\
\hline $\mathrm{CN}$ & Pernambucana IAC 580 & $1,00^{*}$ & 0 & 0 & $11,20 \mathrm{~b}$ \\
\hline $\mathrm{CN}$ & Precoce IAC 281 & $1,00^{*}$ & 0 & 0 & $14,40 \mathrm{~b}$ \\
\hline $\mathrm{CN}$ & Tardia IAC 582 & 0 & 0 & 0 & - \\
\hline $\mathrm{CN}$ & Comum IAC 583 & 0 & 0 & 0 & $16,60 \mathrm{~b}$ \\
\hline $\mathrm{CN}$ & Rio IAC 584 & 0 & 0,66 & 15,42 & $17,60 \mathrm{~b}$ \\
\hline $\mathrm{CN}$ & 114412 IAC 585 & 0 & 0 & 0 & $20,80 \mathrm{a}$ \\
\hline $\mathrm{CN}$ & Córsega IAC 586 & 0 & 0 & 0 & $21,60 \mathrm{a}$ \\
\hline $\mathrm{CN}$ & Céu IAC 587 & 0 & 0 & 0 & $16,00 \mathrm{~b}$ \\
\hline $\mathrm{CN}$ & Paiva IAC 588 & - & 0 & 0 & - \\
\hline $\mathrm{CN}$ & Tardia Sicilia IAC 589 & - & 0 & 0 & - \\
\hline $\mathrm{CN}$ & Seracusa IAC 590 & - & 0 & 0 & - \\
\hline $\mathrm{CN}$ & Tardia IAC 591 & 0 & 0 & 0 & $8,40 \mathrm{~b}$ \\
\hline $\mathrm{CN}$ & Tardia IAC 592 & 0 & 0 & 0 & $4,40 \mathrm{~b}$ \\
\hline $\mathrm{CN}$ & Rio IAC 593 & 0 & 0 & 0 & $12,60 \mathrm{~b}$ \\
\hline $\mathrm{CN}$ & Avana IAC 594 & 0 & 0 & 0 & $23,60 \mathrm{a}$ \\
\hline $\mathrm{CN}$ & Israel IAC 599 & 0 & 0 & 0 & $20,20 \mathrm{a}$ \\
\hline $\mathrm{CN}$ & Paraguaia IAC 600 & 0 & 0 & 0 & - \\
\hline $\mathrm{CN}$ & Umbigo IAC 601 & 0 & 0 & 11,22 & $14,00 \mathrm{~b}$ \\
\hline $\mathrm{CN}$ & Mogi das Cruzes IAC 606 & 0 & 0 & 0 & $23,00 \mathrm{a}$ \\
\hline $\mathrm{CV}$ & Montenegrina & - & 0 & 0 & - \\
\hline $\mathrm{CV}$ & Rainha & - & 0 & 0 & - \\
\hline CV & Tangerina Dancy IAC 188 & $4,00^{* *}$ & 18,40 & 53,22 & - \\
\hline $\mathrm{CN}$ & Tangor Murcott & $4,00^{* *}$ & 9,86 & 78,30 & - \\
\hline CV (\%) & & & & & 33,44 \\
\hline
\end{tabular}

Valores seguidos pela mesma letra pertencem a um mesmo grupo pelo teste Skott-Knott $(\mathrm{p}<0,05) ;{ }^{*}$ menos de $1 \%$ de sintomas em frutos; ${ }^{* *}$ mais de $2,5 \%$ de sintomas em frutos; $\mathrm{CV}$ = clone velho; $\mathrm{CN}=$ clone novo

nível de sintomas nas brotaçóes novas (notas $\leq 1$ = poucos sintomas) (Tabela 1). Esses acessos apresentaram sintomas nos frutos com menos de $1 \%$ de área lesionada, segundo a escala de Renaud et al. (2008), valores esses considerados baixos quando comparados com a tangerina Dancy e o tangor Murcott, que apresentaram nota 4 (valor máximo da escala, com muitos sintomas e seca de brotaçóes novas) e mais de $2,5 \%$ de área lesionada nos frutos, evidenciando que esse grupo de plantas é resistente à doença.

Quando avaliadas in vitro (Tabela 1), em folhas destacadas, com até 48 horas de inoculação com o fungo, apenas um acesso (Rio IAC 584) em estudo apresentou sintomas da doença, sendo que a área de lesão foi inferior $(0,66 \%)$ às áreas foliares afetadas nas variedades suscetíveis (Dancy - 18,4\% e Murcott-9,86\%). Após 72 horas da inoculação in vitro, observou-se maior número de acessos (seis) com sintomas de MMA, sendo esses as mexericas Ipanema IAC 192, Pará IAC 193, Revero IAC 195, Pará IAC 203, Rio IAC 584 e Umbigo IAC 601, dentre os quais, apenas dois (Pará IAC 193 e Revero IAC 195) foram sintomáticos no campo.

Resultados similares foram encontrados por Pacheco et al. (2012) e Souza et al. (2009), cujos estudos demonstraram que algumas tangerinas e híbridos apresentaram sintomas mais severos em folhas destacadas do que nas folhas ou frutos 


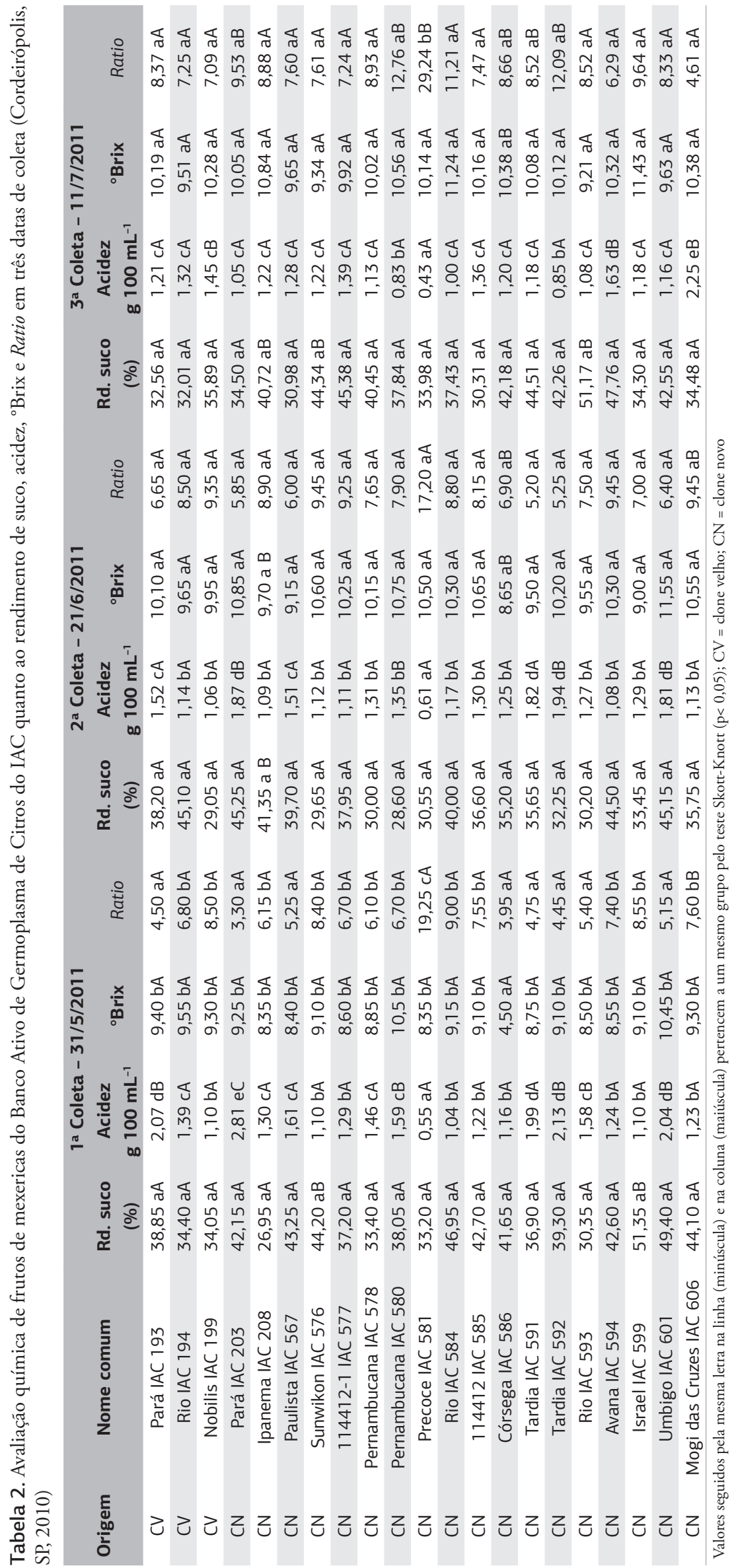


de fruta fresca, embora o mercado brasileiro não seja tão exigente quanto a essa característica.

Com relação à maturação dos frutos (Tabela 2), os resultados evidenciaram que as mexericas Nobilis IAC 199, Precoce IAC 581, Rio IAC 584 e Israel IAC 599 foram mais precoces em relação à variedade padrão (Rio IAC 194). Essas variedades tinham, no mês de maio, valores de Ratio igual ou superior a 8,5 , considerado mínimo para as mexericas no estado de São Paulo (CEAGESP, 2011), enquanto para a mesma época de coleta esse valor foi de 6,8 para a mexerica Rio IAC 194.

Os acessos de mexericas Pará IAC 193, Pará IAC 203, Paulista IAC 567, Pernambucana IAC 578, Pernambucana IAC 580, Córsega IAC 586, Rio IAC 593, Umbigo IAC 601, Tardia IAC 591, Tardia IAC 592, 114412 IAC 577 e 114412 IAC 585 foram mais tardios, sendo ótimas opções para ampliar o período de safra no estado de São Paulo. As demais, Ipanema IAC 208, Sunwikon IAC 576, Avana IAC 594 e Mogi das Cruzes IAC 606, foram consideradas de meia-estação, pelo comportamento semelhante à variedade padrão (Rio IAC 194).

No Rio Grande do Sul, Sartori et al. (2006) relataram como período ideal de maturação, para a mexerica Caí, entre abril e junho e, para a Montenegrina, de agosto a outubro. Para o estado de Sáo Paulo, a tradicional Mexerica do Rio (Rio IAC 194) tem seus frutos apropriados para colheita em campo. Esta constatação pode ser explicada pelo fato de as folhas destacadas apresentarem alteraçôes fisiológicas que podem refletir no seu nível de resistência, tornando-as mais suscetíveis à $A$. alternata e outros microrganismos, uma vez que, ao ser retirada da planta, a folha perde seu poder de resposta à infecção por patógenos (Azevedo et al., 2010; Baudoin e Eckert, 1985; Chutia et al., 2009).

Em geral, foi observada baixa incidência de sintomas e em poucos acessos e, portanto, pode-se afirmar que as variedades do grupo das mexericas são resistentes à Alternaria alternata e constituem boas opçôes de plantio para a citricultura brasileira.

\section{Características físico-químicas dos frutos}

Quanto ao número médio de sementes por fruto, 16 acessos apresentaram um número médio de 15 sementes, semelhante ao da variedade mexerica Rio (Schwarz e Brugnara, 2009), sendo que os menores números, 4,4 e 8,4 sementes por frutos, foram observados, respectivamente, nas mexericas tardias IAC 591 e IAC 592 (Tabela 1). A variedade Montenegrina, que é de produção tardia no Rio Grande do Sul, apresenta de seis a oito sementes por fruto (Schwarz e Brugnara, 2009). Tangerinas apirenas ou com baixo número de sementes são desejadas para o mercado

Tabela 3. Índice de cor da casca dos frutos de acessos de mexericas do Banco Ativo de Germoplasma de Citros obtido em três datas de coleta (Cordeirópolis, SP, 2010)

\begin{tabular}{|c|c|c|c|c|}
\hline \multirow{2}{*}{ Origem } & \multirow{2}{*}{ Nome comum } & \multicolumn{3}{|c|}{ Data de coleta } \\
\hline & & $31 / 5 / 2011$ & $21 / 6 / 2011$ & $11 / 7 / 2011$ \\
\hline $\mathrm{CV}$ & Pará IAC 193 & $-16,54 \mathrm{aA}$ & $-6,01 \mathrm{bB}$ & $-5,04 \mathrm{aB}$ \\
\hline CV & Rio IAC 194 & $-4,77 \mathrm{cA}$ & $1,29 \mathrm{bB}$ & $2,47 \mathrm{cB}$ \\
\hline $\mathrm{CN}$ & Nobilis IAC 199 & $-1,78 \mathrm{dA}$ & $3,29 \mathrm{bB}$ & $4,72 \mathrm{cB}$ \\
\hline $\mathrm{CN}$ & Pará IAC 203 & $-13,74 \mathrm{aA}$ & $1,39 \mathrm{bB}$ & $2,58 \mathrm{cB}$ \\
\hline $\mathrm{CN}$ & Ipanema IAC 208 & $-3,06 \mathrm{cA}$ & 1,94 bB & $4,18 \mathrm{cB}$ \\
\hline $\mathrm{CN}$ & Paulista IAC 567 & $-4,70 \mathrm{cA}$ & $2,06 \mathrm{bB}$ & $2,04 \mathrm{cB}$ \\
\hline $\mathrm{CN}$ & Sunwikon IAC 576 & $0,22 \mathrm{dA}$ & $1,92 \mathrm{bA}$ & $2,84 \mathrm{cA}$ \\
\hline $\mathrm{CN}$ & 114412 IAC 577 & $-1,08 \mathrm{dA}$ & $3,73 \mathrm{bB}$ & $4,71 \mathrm{cB}$ \\
\hline $\mathrm{CN}$ & Pernambucana IAC 578 & $-0,36 \mathrm{dA}$ & $3,13 \mathrm{bB}$ & 4,83 cB \\
\hline $\mathrm{CN}$ & Pernambucana IAC 580 & $0,31 \mathrm{dA}$ & $2,95 \mathrm{bA}$ & $4,89 \mathrm{cA}$ \\
\hline $\mathrm{CN}$ & Precoce IAC 581 & $0,36 \mathrm{dA}$ & 3,17 bA & $4,08 \mathrm{cA}$ \\
\hline $\mathrm{CN}$ & Rio IAC 584 & $-0,18 d A$ & $1,59 \mathrm{bA}$ & $3,47 \mathrm{cA}$ \\
\hline $\mathrm{CN}$ & 114412 IAC 585 & $0,49 \mathrm{dA}$ & $3,04 \mathrm{bA}$ & $4,24 \mathrm{cA}$ \\
\hline $\mathrm{CN}$ & Córsega IAC 586 & $-0,21 d A$ & $2,47 \mathrm{bA}$ & $3,93 \mathrm{cA}$ \\
\hline $\mathrm{CN}$ & Tardia IAC 591 & $-10,31 b A$ & $-2,88 \mathrm{aB}$ & $-0,07$ bB \\
\hline $\mathrm{CN}$ & Tardia IAC 592 & $-9,20 \mathrm{bA}$ & $-6,71$ aA & $-0,04$ bB \\
\hline $\mathrm{CN}$ & Rio IAC 593 & $-2,02 d A$ & $2,22 \mathrm{bB}$ & $3,74 \mathrm{cB}$ \\
\hline $\mathrm{CN}$ & Avana IAC 594 & $1,59 \mathrm{dA}$ & $3,38 \mathrm{bA}$ & $3,75 \mathrm{cA}$ \\
\hline $\mathrm{CN}$ & Israel IAC 599 & $1,01 \mathrm{dA}$ & 3,14 bA & $3,90 \mathrm{cA}$ \\
\hline $\mathrm{CN}$ & Umbigo IAC 601 & $-5,12 c A$ & $4,30 \mathrm{bB}$ & $5,34 \mathrm{cB}$ \\
\hline $\mathrm{CN}$ & Mogi das Cruzes IAC 606 & $-5,03 \mathrm{cA}$ & $0,36 \mathrm{bB}$ & $3,00 \mathrm{cB}$ \\
\hline
\end{tabular}

Valores seguidos pela mesma letra na linha (maiúscula) e na coluna (minúscula) pertencem a um mesmo grupo pelo teste $\mathrm{Skott}-\mathrm{Knott}(\mathrm{p}<0,05) ; \mathrm{CV}=$ clone velho; $\mathrm{CN}=$ clone novo 


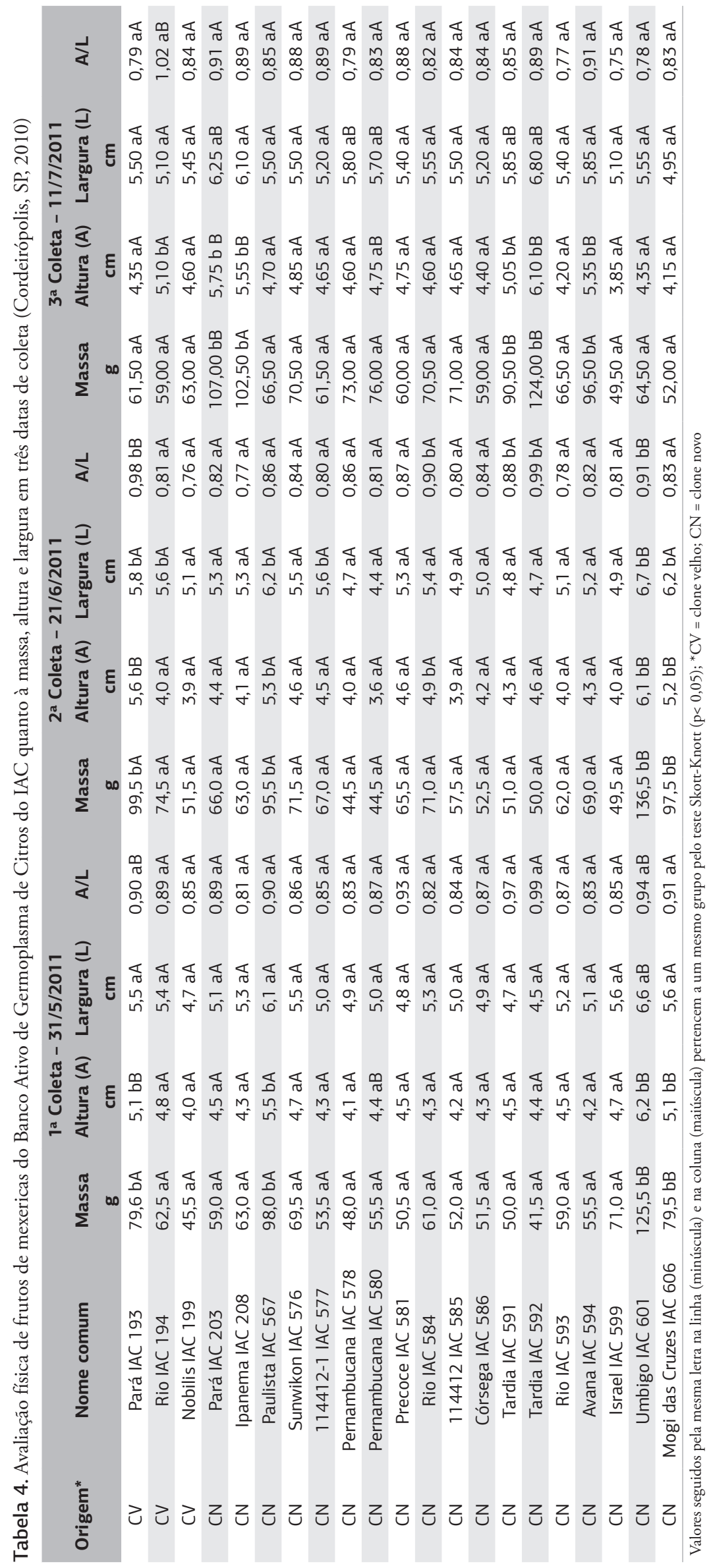


entre os meses de março e julho, com pico de produção entre abril e junho (Pio et al., 2005). Ainda não foi possível avaliar, em São Paulo, a mexerica Montenegrina, pois foi recentemente introduzida no BAG Citros IAC. Essa variedade de mexerica apresenta grande importância econômica no Rio Grande do Sul, por apresentar maturação tardia dos frutos; entretanto os resultados obtidos no presente estudo mostraram que existem outros acessos de mexerica do BAG Citros IAC que podem ser utilizados para ampliar o período de safra de mexerica no estado de São Paulo.

Em geral, todos os acessos estudados apresentaram valores de rendimento de suco apropriados para a comercialização, não havendo diferença estatística entre eles, ou seja, apresentaram valores de rendimento de suco igual ou superior a 35\% (Tabela 2), mínimo exigido para o estado de São Paulo (CEAGESP, 2011).

Em relaçáo à cor da casca foram observados valores de índice muito baixos na primeira data de coleta, em maio, para os acessos de mexerica Pará IAC 193 (-16,54), Pará IAC $203(-13,74)$, Tardia IAC $591(-10,31)$ e Tardia IAC 592 $(-9,20)$, indicando frutos ainda muito verdes (Tabela 3$)$. De fato, essas variedades estão entre aquelas com maturação de frutos mais tardios, a partir de junho (Tabela 2). Por outro lado, as variedades precoces Nobilis IAC 199, Precoce IAC 581, Rio IAC 584 e Israel IAC 599 apresentaram valores de índices mais próximos de zero, o que indica tonalidades verdes alaranjadas, mais apropriadas para a comercialização. Para todas as variedades estudadas observa-se claramente um aumento na intensidade de coloração da casca nas épocas de colheita dos frutos (Tabela 3).

De uma forma geral, os frutos no campo eram frutos pequenos, com massa inferior a $100 \mathrm{~g}$, valor considerado adequado para a comercializaçáo de variedades do grupo das mexericas (Tabela 4). O tamanho reduzido dos frutos, observados na safra 2010/2011, possivelmente deve-se à alternância de produção, característica comum das mexericas, que leva a planta produzir, em safras alternadas, uma grande quantidade de frutos pequenos, pela falta do raleio. Deve ser levado em consideraçáo que o pomar avaliado trata-se de um Banco de Germoplasma, mantido no campo visando a conservação de germoplasma e não a produção comercial de frutas e, portanto, sem os tratos culturais comuns em pomares comerciais.

Neste trabalho, foram selecionados quatro acessos precoces e 11 acessos que foram mais tardios que a variedade padrão, que podem ser boas alternativas para a ampliação do período de safra de mexericas para o estado de Sáo Paulo. Além disso, a evidente resistência das mexericas à mancha marrom de alternária reforça a sua importância na produção comercial de tangerinas, visando a resistência varietal como controle da doença no campo, e as tornam ótimas opçóes para o mercado de frutos de mesa.

\section{CONCLUSÃO}

O grupo das mexericas do Banco de Germoplasma de Citros do IAC apresenta acessos com diferentes épocas de maturação dos frutos e com resistência à mancha marrom de alternária, os quais constituem materiais potenciais para a citricultura paulista, visando a ampliação do período de colheita e o controle varietal da doença no campo.

\section{AGRADECIMENTOS}

Os autores agradecem aos estagiários do CAPTACSM Mayara S. Gonzaga, Rafael A. Rodrigues e Daniel B. Castro, pelo auxílio em alguns dos experimentos que foram conduzidos, e ao $\mathrm{CNPq}$, pelo auxílio financeiro.

\section{REFERÊNCIAS}

AZEVEDO, F.A.; POLYDORO, D.A.; BASTIANEL, M.; KUPPER, K.C.; STUART, R.M.; COSTA, F.P.; PIO, R.M. Resposta de diferentes genótipos de tangerinas e seus híbridos à inoculação in vitro e in vivo de Alternaria alternata. Revista Brasileira de Fruticultura, v.32, p.110, 2010. http://dx.doi.org/10.1590/S0100-29452010005000085

BAUDOIN, A.B.A.M.; ECKERT, J.W. Development of resistance against Geotrichum candidumin lemon peel injuries. Phytopathology, v.75, p.174-179, 1985. http://dx.doi.org/10.1094/Phyto-75-174

COMPANHIA DE ENTREPOSTOS E ARMAZÉNS GERAIS DE SÃO PAULO - CEAGESP. Normas de classificação de citros de mesa. São Paulo: CEAGESP, 2011. 12p.

CHUTIA, M.; BHUYAN, P.D.; PATHAK, M.G.; SARMA, T.C.; BORUAH, P. Antifungal activity and chemical composition of Citrus reticulata Blanco essential oil against phytopathogens from North East India. Food Science and Technology, v.42, p.777-780, 2009.

FERREIRA, D.F. SISVAR: um programa para análises e ensino de estatística. Revista Symposium, v.6, p.36-41, 2008.

INSTITUTO BRASILEIRO DE GEOGRAFIA E ESTATÍSTICA - IBGE. Produção agrícola mundial. Disponível em: <ftp://ftp.ibge.gov.br/Producao_Agricola/Producao_Agricola_ Municipal_[anual]/2011/pam2011.pdf>. Acesso em: 29 jan. 2014.

JIMENEZ-CUESTA, M.; CUQUERELLA C.J.; MARTINEZJAVEGA, J.M. Teoria y practicca de la desverdización de los cítricos. Madrid: INIA, 1983. 22p. (INIA. Hojatécnica, n.46).

PACHECO, C.A.; MARTELLI, I.B.; POLYDORO, D.A.; SCHINOR, E.H.; PIO, R.M.; AZEVEDO, F.A. Resistance and susceptibility of mandarins and hybrids to Alternaria alternata. Scientia Agrícola, v.69, p.386-392, 2012. http://dx.doi.org/10.1590/ S0103-90162012000600007

PERES, N.A.R.; AGOSTINI, J.P.; TIMMER, L.W. Outbreaks of Aternaria brown spot of citrus in Brazil and Argentina. Plant Disease, v.87, p.750, 2003. http://dx.doi.org/10.1094/PDIS.2003.87.6.750C 
PIO, R.M.; FIGUEIREDO, J.O.; STUCHI, E.S.; CARDOSO, S.A.B. Variedades copas. In: MATTOS JUNIOR, D.; NEGRI, J.D.; PIO, R.M.; POMPEU JUNIOR, J. (Org.). Citros. Cordeirópolis: Centro APTA Citros Sylvio Moreira, 2005. p.37-60.

REED, J.B.; HENDRIX, JUNIOR, C.M.; HENDRIX, D.L. Quality control manual for citrus processing plants. Book I, Florida: Intercit Inc. Safety Harbor, 1986. 250p.

RENAUD, M.S.A.; AMORIM, L.; LOURENÇO, S.A.; SPOSITO, M.B. Escala diagramática para avaliação da mancha marrom de alternária de citros. Summa Phytopathologica, v.34, p.270-271, 2008. http:// dx.doi.org/10.1590/S0100-54052008000300015

SARTORI, I.A.; THEISEN, S.; KOLLER, O.C.; REIS, B.; NICHELE, F.S.; LIMA, J.G. Poda e raleio manual de frutos em tangerineiras cv. Montenegrina (Citrus deliciosa Tenore), apreciação econômica. Pesquisa Agropecuária Gaúcha, v.12, p.45-50, 2006.

SCHWARZ, S.F. Melhoramento Genético e variedades. In: KOLLER, O.C. (Ed.). Citricultura, cultura de tangerineiras: tecnologia de produção, pós-colheita e industrialização. Porto Alegre: Editora Rígel, 2009. p.35-48.

SCHWARZ, S.; BRUGNARA, E.C. Classificaçáo Botânica, Morfologia e Fisiologia. In: KOLLER, O.C. (Ed.). Citricultura, cultura de tangerineiras: tecnologia de produção, pós-colheita e industrialização. Porto Alegre: Editora Rígel, 2009. p.35-48.

SCOTT, A.J.; KNOTT, M. A cluster analysis method for grouping means in the analysis of variance. Biometrics, v.30, p.507-512, 1974. http://dx.doi.org/10.2307/2529204

SOUZA, M.C.; STUCHI, E.S.; GOES, A. Evaluation of tangerine hybrid resistance to Alternária alternata. Scientia Horticulturae, v.123, p.1-138, 2009. http://dx.doi.org/10.1016/j.scienta.2009.07.005

STUART, R. M.; BASTIANEL, M.; AZEVEDO, F.A.; MACHADO, M.A. Alternaria brown spot. Laranja, v.30, p.29-44, 2009.

TIMMER, L.W.; PEEVER, T.L.; SOLEIL, Z.; AZUYA, K.; KIMITSU, A. Alternaria diseases of citrus-novel pathosystems. Phytopathologia Mediterranea, v.42, p.99-112, 2003. 